\title{
Study Subject Assessment Time
}

National Cancer Institute

\section{Source}

National Cancer Institute. Study Subject Assessment Time. NCI Thesaurus. Code

C162177.

The time of the subject status assessment. 